\title{
An X-efficiency Analysis of Chinese Commercial Banks Based on Fuzzy DEA Model
}

\author{
Shuangjie Li Dongsheng Zhai Chong Wang Na Wang \\ School of Economics and Management, Beijing University of Technology, Beijing, 100022, P. R. China
}

\begin{abstract}
In this paper the Fuzzy DEA Model was constructed. The model conducted an X-efficiency measure on Chinese Commercial Banks. At last Cause analyses and policy suggestions was given.
\end{abstract}

Keywords: Fuzzy DEA Model, Chinese Commercial Banks

\section{X-efficiency and fuzzy DEA model}

\subsection{X-efficiency}

$\mathrm{X}$-inefficiency is the index measuring intra-enterprise efficiency. The concrete manifestation is low efficiency exists in the enterprise. Namely it is unnecessary to change the quantity or the proportion of input. Only the production organization form needs reasonable change to enhance the production efficiency. This kind of low efficiency is not caused from the unreasonable allocation of input. But its concrete cause is not known yet. Therefore it is called $\mathrm{X}$-inefficiency (is also called $\mathrm{X}$ low-efficiency or $\mathrm{X}$ non-efficiency).

H.Laibinsitan [4] thinks that X-efficiency is the aggregation of all the other efficiencies except the allocation efficiency. Enterprise technical efficiency is only the special cases of the X-efficiency that all the workers has the maximum effort of $100 \%$.

B.Mester [2] utilize X-efficiency theory to discuss the intra-banking microeconomic efficiency, study certain bank's (or some kind of bank's) staff's different choices of effort which decided the efficiency gap compared with the best performance inside the industry, thus obtaining the way to enhance efficiency. M. K. Hassan, and D. R.Tufte, [5] take the Glaimer Bank as example to study the X-efficiency of groupbased credit organization. Y.Altunba, E.P.M.Gardener, P.Molyneux, and B. Moore [1] use the Fourier function form and the stochastic cost frontier approach to estimate the scale efficiency, $\mathrm{X}$ inefficiency and technical progress of a European bank sample in 19891997. D.Leigh and J.B.Hall, [3] analyze the technical efficiency and scale efficiency of a Japanese crossbank's data sample, classify the efficiency analysis from individual bank, bank type and bank scale with the controlling bad loan as an exogenous variable affecting the bank's efficiency.

\subsection{Fuzzy DEA model}

Among many production elements of commercial bank, the main is bank staff. The bank staff directly represented the bank's business level and become the bank's solid foundation. The bank's reflection in customers directly comes from bank staff. Therefore, the performances of bank staff decide the bank efficiency directly.

Usually the bank staff have freedom in diligence decision in certain scope, namely the staff can independently decide the endeavor degree. In fact, the staff tends to provide the proper degree endeavor, while the bank tends to provide the proper work price - wage level. The result of balancing the two sides is neither the lowest point nor the peak of the work and the wages, but is some position in the middle. This is the result of games which the staff and the enterprise. There is an inertia region in the staff endeavor degree. In the graph, sector [e1, e2] is the inertia region.

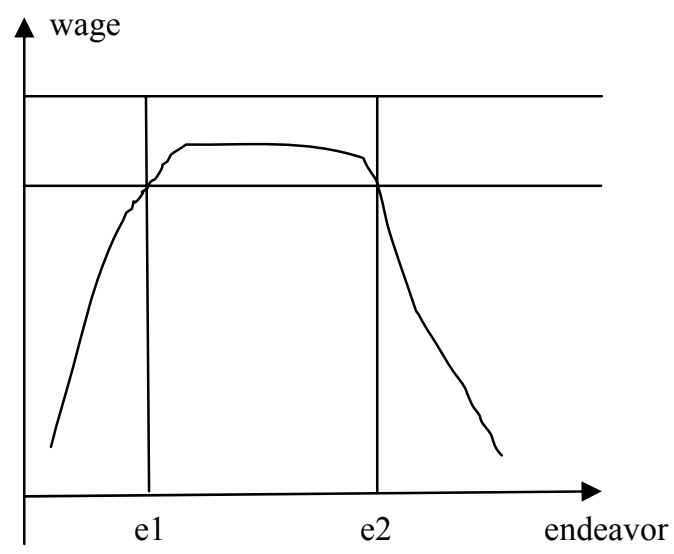

Fig 1: Inertia region. 
The existence of inertia region, causes the restraint labor input condition of mathematical programming question is not precise any longer, but is fuzzy. This makes us introduce the concept of fuzzy optimization and obtain X-efficiency.

We choose constant return on scale DEA model to calculate X-efficiency. Supposing $\mathrm{n}$ banks use $\mathrm{m}$ kinds of inputs to produce s kinds output, regarding certain specific DMU0, its efficiency value is measured by (1):

$$
\begin{aligned}
& \operatorname{Maxz}_{0}=\sum_{r=1}^{s} u_{r} y_{r 0} \\
& \text { s.t. }\left\{\begin{array}{l}
\sum_{r=1}^{s} u_{r} y_{r j}-\sum_{i=1}^{m} v_{i} x_{i j} \leq 0, j=1,2, \ldots, n \\
\sum_{i=1}^{m} v_{i} x_{i 0}=1, u_{r} \geq 0, r=1,2, \ldots, s \\
v_{i} \geq 0, i=1,2, \ldots, m
\end{array}\right. \\
& \operatorname{Maxz}_{0}=\sum_{r=1}^{s} u_{r} y_{r 0} \\
& \text { s.t. }\left\{\begin{array}{l}
\sum_{r=1}^{s} u_{r} y_{r j}-\sum_{i=1}^{m} v_{i} x_{i j} \leq 0, j=1,2, \ldots n \\
\sum_{i=1}^{m} v_{i} x_{i 0}=1 \\
u_{r} \geq 0, r=1,2, \ldots s \\
v_{i} \geq 0, i=1,2, \ldots m
\end{array}\right.
\end{aligned}
$$

Among them, xij represents the jth DMU's quantity of the ith input. yrj represents the jth DMU's quantity of the rth output; Variable ur, vi represent weight.

Now we will carry on fuzzy processing to the model's input variable. Suppose the fuzzy variable is $\tilde{x}_{i j}$, then (1) transform to (2).

Make $\tilde{x}_{i j}$ 's membership function is $\mu$ (xij), supposing $\mu$ (xij) is monotone function. Then xij0 may stand for $\tilde{x}_{i j}$. The replacement is the following model (3) and (4):

$$
\begin{aligned}
& \operatorname{Maxz}_{0}=\sum_{r=1}^{s} u_{r} y_{r 0} \\
& \text { s.t. }\left\{\begin{array}{l}
\sum_{r=1}^{s} u_{r} y_{r j}-\sum_{i=1}^{m} v_{i} x_{i j}{ }^{0} \leq 0, j=1,2, \ldots n \\
\sum_{i=1}^{m} v_{i} x_{i 0}=1 \\
u_{r} \geq 0, r=1,2, \ldots s \\
v_{i} \geq 0, i=1,2, \ldots m
\end{array}\right.
\end{aligned}
$$

$$
\operatorname{Min} \theta_{0}
$$

$$
\text { s.t. }\left\{\begin{array}{l}
\sum_{j=1}^{n} \lambda_{j} y_{r_{j}} \geq y_{r 0}, r=1,2, \ldots . . \\
\theta_{0} x_{i o}-\sum_{j=1}^{n} \lambda_{j} x^{0} \geq 0, i=1,2, \ldots m \\
\lambda_{j} \geq 0, j=1,2, \ldots n
\end{array}\right.
$$

The obtained optimal solution $\theta 0$ is the $\mathrm{X}$ efficiency value.

\section{Data and empirical result}

This article chooses four state-owned commercial banks and ten joint-stock banks of our country in 2000-2005 as samples to research their X-efficiency. We Use "intermediary method" to define bank's inputs and outputs. The input variable is: $\mathrm{X} 1-$ staff number, $\mathrm{X} 2$ - total asset, the output variable is: $\mathrm{Y} 1$ deposit, Y2-loan, Y3- profit before tax.

Data source comes from Chinese statistics yearbook, Chinese financial statistics yearbook, and each bank's annual report.

Based on the inertia theory, individual's degree of effort presents inelastic condition in a certain degree of satisfying scope, namely individual has the nonefficiency state. Then more people are needed to complete the schedule work. Therefore, we take the input variable X1- staff number as fuzzy variable. Because staff number is influenced by bank scale, therefore we take off the influence of scale factor from the staff number membership function, using the unit asset staff number to define the membership function.

Suppose $\alpha=$

$$
\mu\left(\frac{x_{i 1}}{x_{i 2}}\right)=\frac{1-\exp \left[-4 \frac{x_{i 1} / x_{i 2}-\max _{i}\left(x_{i 1} / x_{i 2}\right)}{\hat{x}_{i 1} / x_{i 2}-\max _{i}\left(x_{i 1} / x_{i 2}\right)}\right]}{1-\exp (-4)}
$$


Among them, $\hat{x}_{i 1}$ is the minimum staff number if other factors are invariable, may be obtained through the DEA method.

Then its inverse function is:

$$
\begin{aligned}
x_{i 1}= & \left\{\max _{i}\left(\frac{x_{i 1}}{x_{i 2}}\right)-\right. \\
& \left.(1 / 4)\left(\frac{\hat{x}_{i 1}}{x_{i 2}}-\max _{i}\left(\frac{x_{i 1}}{x_{i 2}}\right)\right) \ln \left[1-\alpha\left(1-e^{-4}\right)\right]\right\} \times x_{i 2}
\end{aligned}
$$

Substituting into the model may obtain linear programming including the membership $a$. When $a$ takes different value, we may solve different linear programming model, obtain optimal solution under different membership.

$$
\begin{aligned}
& \text { Min } \theta_{0} \\
& \text { s.t. }\left\{\begin{array}{l}
\sum_{j=1}^{n} \lambda_{j} y_{r j} \geq y_{r 0}, r=1,2, \ldots s . \\
\theta_{0} x_{i 0}{ }^{0}-\sum_{j=2}^{n} \lambda_{j} x_{i j}-\lambda_{1}\left\{\max _{i}\left(\frac{x_{i 1}}{x_{i 2}}\right)-(1 / 4)\left(\frac{\hat{x}_{i 1}}{x_{i 2}}-\right.\right. \\
\left.\left.\max _{i}\left(\frac{x_{i 1}}{x_{i 2}}\right)\right) \ln \left[1-\alpha\left(1-e^{-4}\right)\right]\right\} \times x_{i 2} \geq 0, i=1,2, . . m \\
\lambda_{j} \geq 0, j=1,2, . . n
\end{array}\right.
\end{aligned}
$$

\begin{tabular}{c|l|l|l|l|l|l}
\hline bank & $\mathrm{a}=0.9$ & 0.7 & 0.5 & 0.3 & 0.1 & TE \\
\hline 1 & 0.998 & 0.998 & 0.998 & 0.998 & 0.998 & 0.998 \\
\hline 2 & 0.964 & 0.962 & 0.96 & 0.96 & 0.959 & 0.959 \\
\hline 3 & 1 & 1 & 1 & 0.982 & 0.968 & 1 \\
\hline 4 & 1 & 1 & 1 & 1 & 1 & 1 \\
\hline 5 & 1 & 1 & 1 & 1 & 1 & 1 \\
\hline 6 & 1 & 0.995 & 0.976 & 0.964 & 0.957 & 1 \\
\hline 7 & 1 & 1 & 1 & 1 & 1 & 1 \\
\hline 8 & 0.979 & 0.977 & 0.976 & 0.976 & 0.976 & 0.975 \\
\hline 9 & 1 & 1 & 1 & 1 & 1 & 1 \\
\hline 10 & 1 & 1 & 1 & 1 & 1 & 1 \\
\hline 11 & 0.989 & 0.989 & 0.989 & 0.989 & 0.989 & 0.988 \\
\hline 12 & 0.967 & 0.965 & 0.963 & 0.963 & 0.962 & 0.962 \\
\hline 13 & 0.906 & 0.892 & 0.876 & 0.864 & 0.855 & 0.921 \\
\hline 14 & 1 & 1 & 1 & 1 & 1 & 1 \\
\hline \multicolumn{7}{|l|}{ Table $1:$ Efficiency result } \\
\end{tabular}

Table 1: Efficiency result.

Using software DEAP to obtain the result (2005).

The table also lists the technical efficiency value calculated by the model. Therefore we can judge the ideal degree of staff works by the listed X-efficiency value.
For example, Min Sheng Bank, the technical efficiency is higher than X-efficiency value when membership is 0.9 , therefore, in this bank; staff member's degree of effort is very high. We can see from the table that the state-owned commercial bank and Guang Fa, Guang Da banks' efficiency is not high.

\section{Cause analyses and suggestions}

\subsection{Cause analyses}

$\mathrm{X}$-efficiency theory believes that the enterprise is composed by different-interest groups, while differentinterest groups are composed by different individuals. In enterprise, because individual has choice rational, exists psychological inertia region, and different individual pursues different interest, therefore it causes $\mathrm{X}$ inefficiency.

\subsubsection{Inertia factor}

Because of the existence of inertia region, when individual's degree of effort relatively fixes in a steady state, he tend to maintain the degree of effort invariable. At this time, subtle change is unable to change individual's degree of effort. Because once it changes, he will continue to maintain the same degree of effort in quite a long time, which will be a large cost to the subtle change. Therefore, this is a chronic disease to X low-efficiency.

At the same time, the existence of inertia region theory also makes people prefer to hesitate to move forward. Because compared with making change, following the old system still is the most effort saving and the most relaxing way. To the enterprises that need to reform and develop, this is the mortal wound without doubt. It is the main reason for enterprises' $\mathrm{X}$ low-efficiency.

\subsubsection{Environmental factors}

Environmental factor also has important influence on individual and the whole $\mathrm{X}$ non-efficiency. Pressures from boss and colleagues and the influence of soft environment like enterprise culture and so on, are all the conditions forming X-inefficiency.

Firstly, boss's pressure, namely after completing the task, whether receiving boss's praise because splendidly completing the task or receiving boss's criticism because completing not well, can affect the staff's quality pursue of the task. Moreover, boss's attitude, namely the tolerant attitude to the staff -only reward and no punishment, or the strict attitude-- only punishment and no reward, or reward and punishment 
are distinct, will affect staff's degree of effort, thus affecting X-efficiency.

Next, colleague's pressure, can also affect the determination of individual's degree of effort. In fact, fellow colleagues' degree of effort in the same position affects each other. After increasingly run and adjustment, each person's degree of effort may be the same as others', in order to avoid outstanding. Then the whole will form an inertia region space, in which each person's degree of effort occurs simultaneously. New member's appearance or some member's withdrawal will possibly affect this region. It will adjust further, achieve a stable condition and continue to the next new changeable factor's appearance.

The enterprise culture is based on the boss and colleague's environment, but it also has a more formidable strength. The enterprise culture have certain compulsory force after forming, namely each person will adjust his own degree of effort to adapt the enterprise with the enterprise culture standard, conform individual's goal to enterprise's goal, form the common value. That will make the members have common value orientation and faiths pursue, but not adjust the enterprise culture with their own standard. Therefore, the enterprise culture also has important influence on the determination of staff's degree of effort.

\subsubsection{Management factors}

The enterprise's internal management level also influence X-efficiency.

(1) Whether the responsibility is clear can affect $\mathrm{X}$-efficiency. That the responsibility is not clear will cause relative departments shifting responsibility onto others and wrangling, irresponsible, playing the edge ball in the work process. Power and responsibility are not well defined, either nobody is willing to do, or the repetition work, which causes nobody is responsible for the problem, unable to solve, and waste of resources and $\mathrm{X}$ low-efficiency.

(2) Because of the organizational structure problem, the enterprise is unable to arouse each person's enthusiasm. To arouse staff's enthusiasm, enterprise's organizational structure must be established reasonably, restrained moderately, and has centralization as well as divided power. If organizational structure is redundant, the information will pass on slowly, the tail greatly does not fall. At the same time, the redundancy of organizational structure is liable to form bureaucratic way of work, which carries out work according to the inherent procedure, cannot fully excavate the internal potential, causing X low-efficiency.

(3) Whether the regulation construction is perfect can also affects staff's enthusiasm. The regulation is not perfect, adopts manageable way by people-ruling primarily, non- fair will inevitably exist because of person's subjectivity. Non- fair causing staff's enthusiasm drop is natural.

In brief, the reason of $\mathrm{X}$ low-efficiency mainly comes from the inconsistency between each person's behavior goals. The economic research must go deep into individuals, must discuss person's subjective initiative. It is very similar with the new-system economics on this point, which emphasize the establishment of incentive and restraint mechanism to make the staff inside the enterprise fully play their roles.

\subsection{Suggestions}

The banking industry is a service industry, providing capital support and circulation for economical movement and enterprise operation, bringing vigor to economic activity. As a service industry, its most important participant and the main body are the bank staff. To enhance the commercial bank's X-efficiency, enhancing staff's degree of effort is the key.

In view of this problem, it is need to pay attention to the following points:

1. Human resources management. Including establishment of personnel employment, existing staff's incentive, and staff's withdrawal mechanism. Choosing new personnel with strict quality condition, taking thorough research on the staff who is going to enter, choosing personnel with high individual inertia region. At the same time, establishing good incentive mechanism to existing staff, playing individual's subjective initiative as far as possible to enhance enterprise's X-efficiency.

Based on the characteristics of our country's commercial banks, the following aspects should be included:

(1) Establish reasonable salary system, stimulate staff's creative value. Fix the staff grade and class of staff according to given system. It is advantageous to the staff to find their own difference, thus stimulating the staff improving work attitude and method and obtaining good performance.

(2) Carry on reasonable disposition to human resources. Design work process reasonable, carry on process reproduction, formulate reasonable labor plan and scientific work responsibility distribution, make the best possible use of men and things, and put an end to that some staff are not competent to the position or some are not fully used. 
(3) Establish achievements examination mechanism, strengthen achievements examination consciousness.

(4) Try hard to establish good physical conditions and humanities environment, pay attention to staff's humanities concern and enterprise culture's identity and fusion, form good work atmosphere, form good influence on staff's degree of satisfaction and loyalty, drive staff's working enthusiasm and power.

2. Enterprise culture. Persist in depending upon the enterprise culture to condense thought, stimulate enthusiasm, and standardize behavior, put forth effort to construct enterprise culture with the enterprise characteristics and conforming to the time requirement, as well as colorful and with bright personality.

(1) Take strengthening the enterprise manageable idea as the key to cultivate enterprise spirit.

(2) Take strengthening the enterprise prestige as the key to portray enterprise image.

(3) Take improving the staff quality as the key to strengthen the staff troop construction.

Through the above several measures, we may change staff's spirit state and behavior custom, fully stimulate the staff, thus enhance staff's degree of effort to enhance X-efficiency. This can inject new vigor to our country's state-owned commercial banks' rapid development, enhance its comprehensive competitive ability, and occupy the initiative better in the increasingly keen competition.

\section{Acknowledgement}

This work is supported by Major Program of the National Natural Science Foundation of China (70639002), Beijing Municipal Natural Science Foundation (9052002), Beijing Municipal Social Science Planning Office and Beijing Municipal Commission of Education (SZ200510005001).

\section{References}

[1] Y. Altunba, E.P.M. Gardener, P. Molyneux, and B. Moore. Efficiency in European banking. European Economic Review, 45(10):1931-1955, December 2001.

[2] B. Mester. Inside the Black Box: What Explains Differences in the Efficiencies of Financial Institutions?. Journal of Banking and Finance, 21:895-947,1997

[3] D. Leigh and J.B. Hall, Maximilian. Efficiency in Japanese banking: An empirical analysis. Journal of Banking and Finance, 27(5): 891-917, May 2003.
[4] H. Leibenstein. Allocative Efficiency Vs. 'XEfficiency'. American Economic Review, 56(3):392-414, 1966.

[5] M. K. Hassan, and D. R.Tufte, The X-Efficiency of a Group-Based Lending Institution: The Case of the Grameen Bank. World Development, 29(6):1071-1082, 2001 